\title{
EstGeoMag: Integrando soluções de Hardware, Software e Internet das Coisas na medição de grandezas Geomagnéticas
}

\author{
Fábio Franco de Oliveira ${ }^{1}$, Rodolfo da Silva Villaça ${ }^{1}$, Celso Alberto Saibel Santos ${ }^{1}$ \\ Roberto Colistete Junior ${ }^{2}$,
}

1Programa de Pós Graduação em Informática (PPGI)

Universidade Federal do Espírito Santo (UFES)

Vitória - ES - Brasil

${ }^{2}$ Departamento de Química e Física

Universidade Federal do Espírito Santo (UFES)

Alegre - ES - Brasil

fabioti6@gmail.com, rodolfo.villacadufes.br, saibel@inf.ufes.br,
roberto.colistetedufes.br

Abstract. This paper presents EstGeoMag, a low cost hardware and software solution, based on concepts found in the Internet of Things, whose main objective is to help obtain geomagnetic quantities with high resolution and precision. In addition to detailing the hardware and software architecture and to evaluate the precision and accuracy of measurements, 105h of the equipment's use were used for the monitoring in the city of Venda Nova do Imigrante, in the state of Espirito Santo, Brazil. The results of these measurements were compared with different high precision commercial magnetometers models used by INPE.

Resumo. Este artigo apresenta o EstGeoMag, uma solução de hardware e software de baixo custo, baseado em conceitos presentes na Internet das Coisas, e que tem como objetivo principal auxiliar a obtenção de grandezas geomagnéticas com alta resolução e precisão. Além do detalhamento da arquitetura em hardware e software, para a avaliação da precisão e acurácia das medidas foram monitoradas $105 \mathrm{~h}$ de uso do equipamento na cidade de Venda Nova do Imigrante, interior do estado do Espírito Santo. Os resultados dessas medições foram comparados com diferentes modelos de magnetômetros comerciais de alta precisão usados pelo INPE. 


\section{Introdução}

O campo magnético ao redor do planeta Terra é gerado pelo efeito de seu núcleo, composto por metal em forma líquida e temperaturas na ordem de $6000^{\circ} \mathrm{C}$. Este campo que se estende por milhares de quilômetros acima da atmosfera é denominado Magnetosfera Terrestre. Ao redor deste campo, nosso planeta é exposto à radiação solar conhecida como "ventos solares". Estes, por sua vez, de acordo com o seu grau de intensidade, podem provocar fenômenos conhecidos como "tempestades solares", que podem causar desde oscilações de tensão em linhas de transmissão de energia elétrica até interferências na comunicação de dados via satélite.

Para o estudo e monitoramento das grandezas físicas relacionadas a estes fenômenos, além do estudo de diferentes tipos de formações rochosas e minerais magnéticos, geólogos e estudantes de geologia de escolas e universidades ao redor do mundo utilizam equipamentos chamados magnetômetros.

Existem diversos tipos de magnetômetros. Eles podem ser construídos a partir de diferentes sensores e tecnologias de acordo com o grau de resolução, precisão e acurácia pretendidos nas medidas. Dentre estes, podem ser destacados (CAMPBELL, 2003): (i) os magnetos-resistivos, de baixo custo e com menor grau de precisão, muito usados em smartphones e tablets; (ii) os de precessão de prótons, mais precisos, porém com maiores custos de manutenção e complexidade na sua utilização e (iii) SQUID, com alto nível de precisão, porém de aquisição e construção restritos devido à alta complexidade de sua operação e custo elevado. Existe ainda um quarto tipo de Magnetômetro que possui um bom nível de precisão e custo mais acessível: o do tipo Fluxgate (CAMPBELL, 2003). Este modelo é usado frequentemente no meio acadêmico e em equipamentos comerciais devido à relativa facilidade de produção em larga escala, custo mais acessível e ótimo nível de precisão para a maioria das aplicações (WANG, 2015).

Um problema em comum entre estes equipamentos, no entanto, é que a implementação de sua arquitetura, tanto de hardware quanto de software, frequentemente é fechada, proprietária, dependente do fabricante. Este fato termina aumentando em demasia o seu custo, sobretudo quando altos níveis de precisão e baixo ruído de medição são requeridos. Como é típico também no cenário acadêmico, há também uma grande dificuldade na integração de soluções computacionais para processamento dos dados de medição devido ao fato de que os fabricantes não compartilham entre si detalhes de sua arquitetura e as ferramentas de aquisição de dados também serem proprietárias e específicas de cada equipamento.

Oliveira et al. (2016) apresentaram um protótipo de uma estação de medição geomagnética, com uso de um único sensor Fluxgate. A arquitetura apresentada é aderente ao modelo de Internet das Coisas. Em sua avaliação, foi verificado que com a funcionalidade de interface Web e o compartilhamento de dados abertos entre os usuários da estação proposta, a plataforma tinha um grande potencial para a aprendizagem de estudantes dos cursos de Geologia e Física. 
No trabalho atual, apresentamos a implementação de uma estação geomagnética completa, de alta resolução e precisão nas leituras das variações do campo geomagnético da Terra, com a leitura de três componentes magnéticas (norte-sul, lesteoeste e vertical) e uso de três sensores Fluxgate. Além disso, a estação, denominada EstGeoMag, possui dois sensores de temperatura para auxílio no monitoramento do ambiente durante a etapa de calibração das medições e uso de GPS para aquisição de informações sobre a localização exata do equipamento no instante das medidas. Com o baixo nível de ruído obtido na sua construção, o EstGeoMag torna-se equiparável aos magnetômetros comerciais de maior custo em uso por organizações privadas ou órgãos públicos, tais como o Instituto Nacional de Pesquisa Espacial (INPE), conforme será mostrado nas seções finais do artigo.

Além da arquitetura aberta de implementação, o EstGeoMag apresenta alguns outros diferenciais: (i) a ideia de computação pervasiva, embarcada com soluções de hardware e software, que auxiliam o usuário desde a calibração e instalação do equipamento, com a análise em tempo real dos dados medidos e o cálculo do magnetismo esperado no local, baseando-se nos modelos teóricos $\mathrm{WMM}^{1}$ (World Magnetic Models) e IGRF $^{2}$ (International Geomagnetic Reference Field); (ii) o processamento dos dados das medições, com o calculo de médias móveis (SMITH, 1997) e (iii) a disponibilização dos dados na interface Web. Essa interface, por sua vez, contém recursos importantes, tais como o gerenciamento do equipamento à distância, a exportação de dados em formato aberto e a visualização das médias das leituras dos sensores por intermédio de gráficos.

Além do detalhamento da arquitetura de hardware e software do EstGeoMag, para a avaliação da precisão e acurácia das medidas, o equipamento foi utilizado por um total de 105 horas na cidade de Venda Nova do Imigrante, no interior do Espírito Santo. Os resultados obtidos foram compatíveis com diferentes magnetômetros comerciais de alta precisão situados em outros estados do território brasileiro.

O restante deste artigo está dividido da seguinte forma: Na Seção 2, serão apresentados e discutidos alguns trabalhos relacionados. Na Seção 3, será apresentada a arquitetura, as características e os desafios encontrados na implementação do EstGeoMag. Na Seção 4, será detalhada a avaliação do EstGeoMag e comparação dos resultados com outros equipamentos comerciais. A Seção 5, conclui o artigo e faz indicações de trabalhos futuros.

\section{Trabalhos Relacionados}

Com o avanço e popularização dos chamados "Computadores de Placa Única" (SBC, ou Single Board Computers), novas soluções integrando hardware e software para fins de coleta de dados de sensores em diferentes áreas do conhecimento, tais como SmartGrids, agricultura e estudos de geologia e geofísica, têm surgido.

Silva Junior (2015) desenvolveu um magnetômetro do tipo Fluxgate com alta sensibilidade para medir campos magnéticos de baixa intensidade. Com o uso de dois

1 https://www.ngdc.noaa.gov/geomag/WMM

2 https://www.ngdc.noaa.gov/IAGA/vmod/igrf.html 
sensores para as componentes magnéticas, o equipamento faz uso de um computador de mesa com o software LabView ${ }^{3}$ para a coleta de dados e de software feito em linguagem Java para a visualização e processamento dos dados. No EstGeoMag, são utilizados 3 sensores para as componentes do campo magnético terrestre e é utilizado software aberto para aquisição dos dados.

Beggan e Marple (2016) propuseram um magnetômetro de baixo custo que usa um SBC, para a coleta e processamento de dados de três sensores do tipo Fluxgate com uso de um ADC (Analog-Digital Converter) de 17 bits. No projeto, foram efetuadas medidas a cada $5 \mathrm{~s}$ e cálculo de médias móveis a cada $10 \mathrm{~min}$. Foi mantido, também, um termômetro externo ao projeto para monitoramento do ambiente e viabilizar a correção dos dados em uma etapa de pós-processamento. Por sua vez, após serem processados os dados são enviados via protocolo RSYNC ou upload para um servidor Web.

Um diferencial de nosso trabalho foi a realização de uma análise de três diferentes tipos de conversores ADC na arquitetura, de acordo com a necessidade de custo versus precisão do usuário da plataforma.

Como nos outros trabalhos encontrados na literatura há sempre a dependência de algum computador externo, seja para o processamento, seja para a disponibilização e acesso aos dados, um diferencial da arquitetura proposta neste artigo é a sua aderência ao modelo de Internet das Coisas. A arquitetura inclui um servidor Web em execução no SBC, oferecendo o acesso remoto aos dados coletados pelo magnetômetro. Além dos dados poderem ser disponibilizados rapidamente na Internet, sem a necessidade de infraestrutura pré-existente, há a possibilidade de uso offline, por meio da rede local sem fio, com acesso direto à interface Web via navegador Web a partir dos computadores e celulares.

\section{Arquitetura de Hardware e Software}

\subsection{Hardware}

A arquitetura de hardware do EstGeoMag é composta pelos módulos representados pela unidades tracejadas na Figura 1. As descrições dos módulos e das suas funcionalidades são as seguintes:

- Unidade 1 - Coleta de Dados: responsável pela coleta dos dados, cujas funções são desempenhadas pelos sensores FluxGate, que são alimentados por um Regulador de Tensão e monitorados por um Sensor de temperatura

- Unidade 2 - Processamento dos Dados: responsável pelo processamento dos dados, cujas funções são desempenhadas pelo Computador de Placa Única com o auxílio de um RTC (Real Time Clock), GPS e um ADC, sendo este último alimentado por outro Regulador de Tensão e também monitorado por mais um sensor de temperatura.

3 http://www.ni.com/labview/pt 


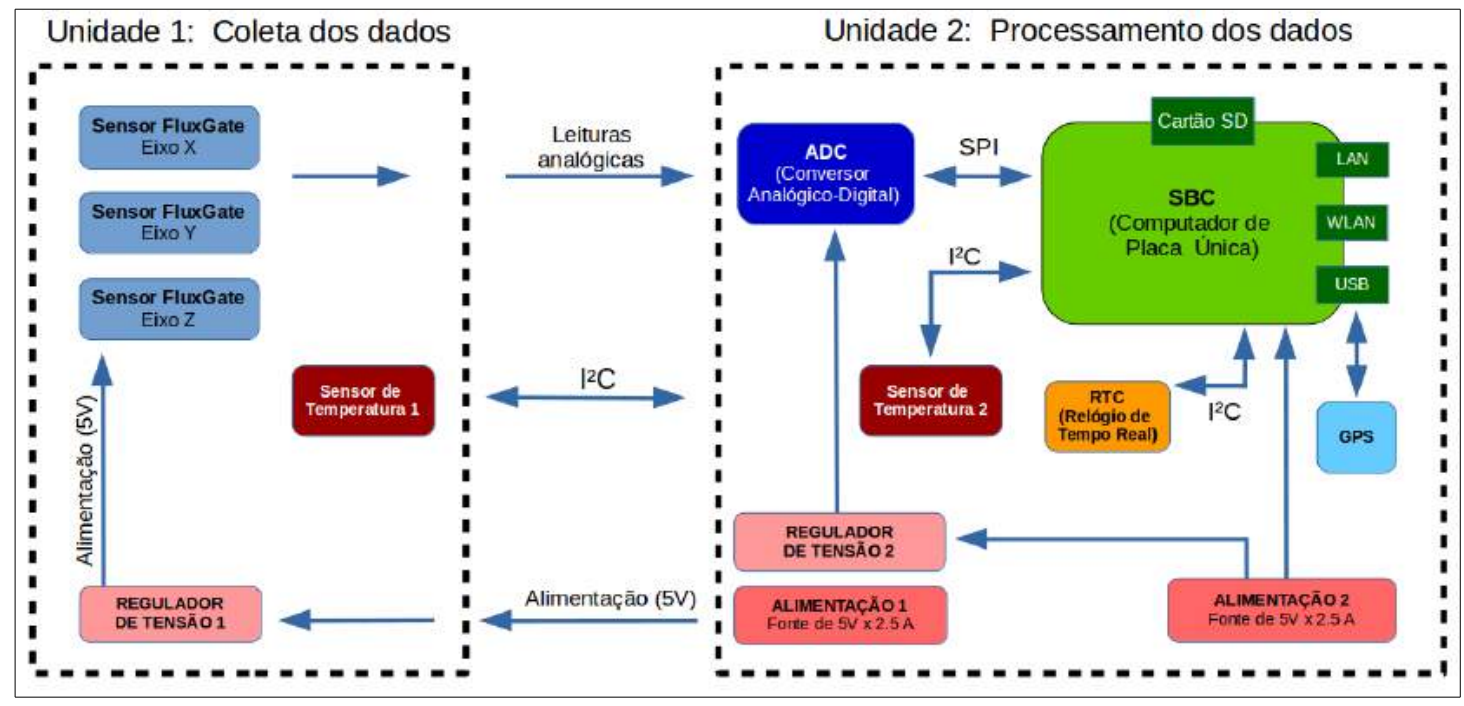

Figura 1: Arquitetura do EstGeoMag

No projeto do EstGeoMag são usados 3 sensores magnéticos do tipo FluxGate para acompanhamento das variações do campo magnético terrestre em três eixos: Norte-Sul, Leste-Oeste e Vertical. Essas variações são associadas a um sinal elétrico gerado nos sensores, medido em Volts e proporcional ao campo magnético medido. Este sinal, de representação analógica, é lido por um ADC responsável pela quantização e digitalização dos valores lidos. O ADC então entrega os dados, por intermédio de um barramento SPI (Serial Peripheral Interface), para o Computador de Placa Única que executa um sistema operacional embarcado de código-fonte aberto. Os sensores de temperatura são conectados por meio de um barramento $\mathrm{I}^{2} \mathrm{C}$ (Circuito Inter-Integrado) e possuem a função de monitorar variações de temperatura nos sensores FluxGate e no ADC no momento da leitura e conversão.

Apesar da curva de transferência da variação do campo magnético em Volts do sensor FluxGate utilizado ser linear, ela é afetada pela temperatura, que também afeta a resposta do regulador de tensão usado em sua alimentação. De maneira similar, também há influência da temperatura do local onde é feita a conversão $\mathrm{AD}$ das medidas realizadas pelo ADC. Por este motivo, a medição de temperatura é necessária para que seja possível compensar a variação (drift) de temperatura nos componentes mais sensíveis do circuito. Desta forma, para aumentar a precisão e acurácia da medição são usados dois sensores de temperatura: um na unidade de coleta, para monitorar o ambiente dos sensores de magnetismo, e outro na unidade de processamento para monitorar o ambiente no momento da conversão AD. 
Duas fontes independentes de $5 \mathrm{~V}$ foram utilizadas no projeto para assegurar uma mínima variação na estabilização da alimentação de energia do EstGeoMag. Considerando que a carga de consumo gerada pelo Computador de Placa Única oscila de maneira significativa. Esta oscilação poderia influenciar na leitura dos sensores de magnetismo em função do fato de que os valores de tensão gerados devido o campo magnético são obtidos de forma relativa à tensão de alimentação dos sensores. Desta forma, se a alimentação dos sensores variar, o resultado da conversão ADC também vai variar de modo proporcional, reduzindo a precisão e a acurácia das medições do EstGeoMag.

Para não haver aumento de temperatura e nem possíveis interferências (ruídos) causados pela própria fonte que alimenta a unidade de coleta de dados, ela é mantida no mesmo involucro da unidade de processamento dos dados e reguladores de tensão foram adicionados em duas placas de circuito impresso personalizadas para complementar o papel de regulação das fontes. Para assegurar precisão no registro do instante exato (data e hora) das medições, é usado também um módulo de Relógio de Tempo Real (RTC), regulado por cristal de quartzo, com compensação de variação de temperatura e alimentação por bateria externa, fazendo com que o sistema mantenha seu horário sempre apurado mesmo em caso de interrupções na alimentação do equipamento.

Complementando o conjunto, foi incluso um módulo de GPS USB de baixo custo para registro das coordenadas do local de instalação do magnetômetro. Os principais componentes utilizados na implementação do EstGeoMag são detalhados na Tabela 1:

Tabela 1. Componentes principais do EstGeoMag

\begin{tabular}{|c|c|}
\hline Sensor de magnetismo FLC-100 & $\begin{array}{l}\text { Fabricante: Stefan Mayer Instruments } \\
\text { Tipo de saída: tensão proporcional ao campo magnético detectado } \\
\text { Faixa de medição: } \pm 100 \mathrm{uT} \\
\text { Tensão de operação: } 5 \mathrm{~V} \pm 5 \% \text { / Consumo: } \sim 2 \mathrm{~mA} \\
\text { Ruído: <3nTpp }(0,1 \text { a } 10 \mathrm{~Hz}) \\
\text { Temperatura de operação: }-40 \text { a } 85^{\circ} \mathrm{C}\end{array}$ \\
\hline Sensor de Temperatura MCP9808 & $\begin{array}{l}\text { Fabricante: Adafruit / Microchip } \\
\text { Resolução: } 0,0625^{\circ} \mathrm{C} / \text { Acurácia: } \pm 0,25^{\circ} \mathrm{C} \\
\text { Faixa de leitura: }-40^{\circ} \mathrm{C} \text { a } 125^{\circ} \mathrm{C} \\
\text { Alimentação: } 2,7 \text { a } 5 \mathrm{~V}\end{array}$ \\
\hline $\begin{array}{l}\text { ADC High-Precision AD/DA } \\
\text { Expansion Board }\end{array}$ & $\begin{array}{l}\text { Fabricante: Waveshare } \\
\text { Resolução: } 8 \text { canais de } 24 \text { Bits } \\
\text { Referência de tensão: } 2,5 \mathrm{~V} \\
\text { Tensão de operação: } 3,3 \text { a } 5 \mathrm{~V} \\
\text { Taxa de amostragem } 2,5 \text { - } 30000 \text { amostras por segundo / Interface: SPI }\end{array}$ \\
\hline $\begin{array}{l}\text { Computador de Placa Única } \\
\text { Raspberry Pi } 3\end{array}$ & $\begin{array}{l}\text { Fabricante: Raspberry Foundation } \\
\text { SoC (System on a Chip): Broadcom BCM2837 } \\
\text { CPU/Memória: } 1.2 \mathrm{GHz} 64 \text { bits quadcore ARMv8 /1024Mb SDRAM } \\
\text { Rede: Ethernet 10/100 / Wireless: } 2.4 \mathrm{GHz} 802.11 \mathrm{n} \\
\text { Alimentação: 5V com consumo de } 700 \mathrm{~mA}(3.5 \mathrm{~W}) \\
\text { Armazenamento: Micro SD }\end{array}$ \\
\hline
\end{tabular}


Na Figura 2 é mostrada uma foto do equipamento com detalhes para o interior das unidades de coleta e a de processamento.

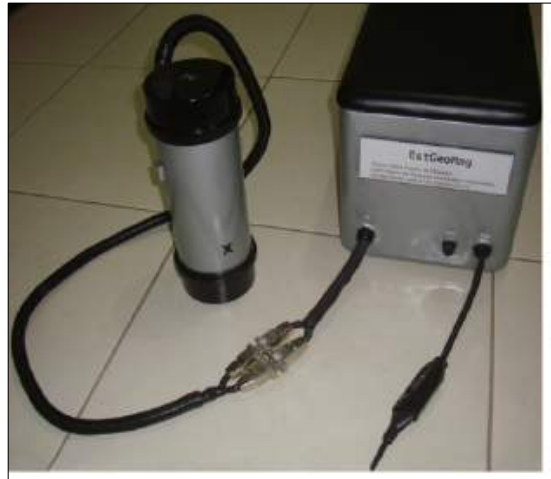

a) Estaçāo pronta para uso

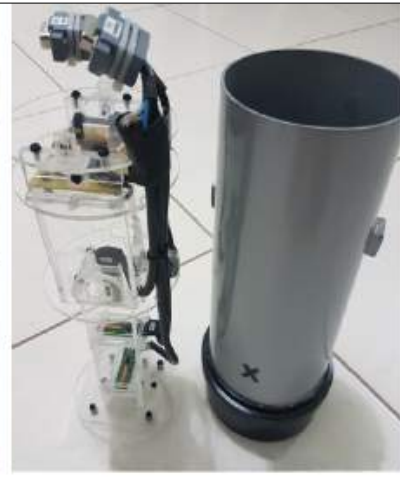

b) Unidade de Coleta dos Dados

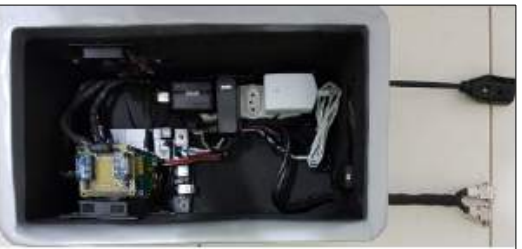

e) Unidade de processamento dos dados

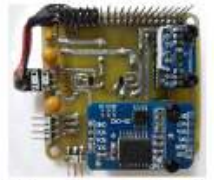

c) Regulador de Tensấ Sensor Temp, RTC Unid. Processamento

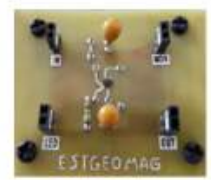

d) Regulador de Tensào Unidade de Coleta

Figura 2: Componentes do EstGeoMag

\subsection{Software}

Com relação ao software embarcado, ele é composto por um sistema operacional de código fonte aberto (Linux), um banco de dados SQL para armazenagem dos dados provenientes das medições, uma aplicação em linguagem Python para processamento das amostras obtidas pelo ADC e um servidor Web para disponibilização remota dos dados. A Figura 3 resume as entradas e saídas de dados, assim como as principais etapas de processamento realizados pelos componentes da arquitetura de software do EstGeoMag.

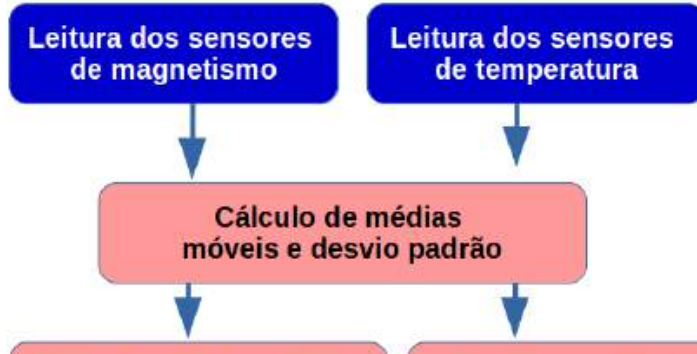

Leitura do módulo de GPS

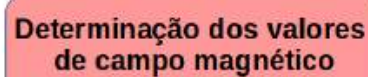
de campo magnético

Compensação por variação de temperatura

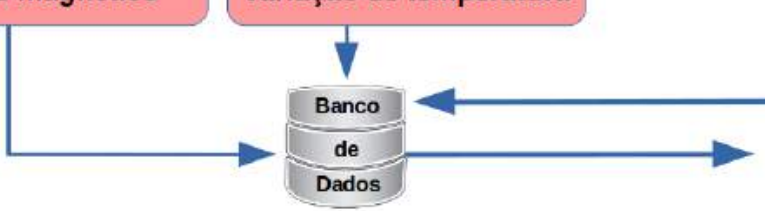

Interface Web com os valores e gráficos

Figura 3: Estrutura do software do EstGeoMag 
Conforme pode ser visto na Figura 3, após serem efetuadas as leituras de temperatura e campo magnético, e após a conversão AD dos dados lidos, um pósprocessamento dos dados é realizado com a finalidade de se aumentar a precisão da conversão e eliminar ruídos aleatórios gerados por outliers (SMITH, 1997). Esse pósprocessamento é feito com a aplicação de médias móveis a uma taxa de 18 medidas por minuto. A escolha desta taxa é justificada pela observação de que efetivamente tem-se pouca variação de amplitude no campo magnético terrestre (da ordem de 1nT, ou menor) entre durante o intervalo de $1 \mathrm{~min}$ e cada sequencia de leitura dos canais usados no ADC levando cerca de 3,2s na configuração atual. Além disto, é calculado e registrado, em tempo real, o desvio padrão das médias das leituras.

Caso seja desejado pelo usuário, o EstGeoMag pode ser reprogramado para trabalhar com médias móveis em um intervalo maior (por exemplo, 180 elementos a cada 10min). Com isso, seria possível obter-se ainda mais precisão, mas haveria o risco de se registrar uma quantidade menor de variações legítimas (sem ruído) durante este intervalo.

A partir do registro das coordenadas de GPS, baseando-se nos modelos teóricos WMM e IGRF, é calculado o valor absoluto do campo magnético previsto para a localidade. Com isso, o pesquisador responsável pode ter uma ideia do grau de distorção entre o valor medido e o valor esperado, o que pode indicar, por exemplo, a existência de interferências geradas por campos magnéticos externos, tais como linhas de alta tensão, ou até mesmo a existência de construções ou ferragens no local pretendido para instalação do EstGeoMag.

Em seguida, a data e horário das medições, os valores de magnetismo e temperatura dos sensores, as coordenadas de altitude, latitude e longitude do GPS, e também os valores calculados pelos modelos teóricos são armazenados no banco de dados SQL em um cartão MicroSD externo de 32gb. O SGBD escolhido, o SQLite versão 3.8.7.1, possui boa versatilidade para uso como software embarcado. Além disto, todos os dados também são salvos simultaneamente no formato aberto de texto delimitado por vírgulas (CSV, ou Comma-Separated Values).

Após terem sido armazenados, os dados podem ser acessados por intermédio da interface Web via rede local ou Internet. Concebida com uma técnica de design Web responsivo, permite a exibição de maneira personalizada na tela de dispositivos como celulares, tablets ou laptops. Os dados e os gráficos das leituras dos sensores de temperatura e de magnetismo são atualizados automaticamente, em tempo real, e exibidos de forma contínua sem que para isso seja preciso a intervenção do usuário. 
A Figura 4 ilustra um exemplo do uso da interface Web em um smartphone para monitoramento das três componentes magnéticas (norte, leste e vertical).

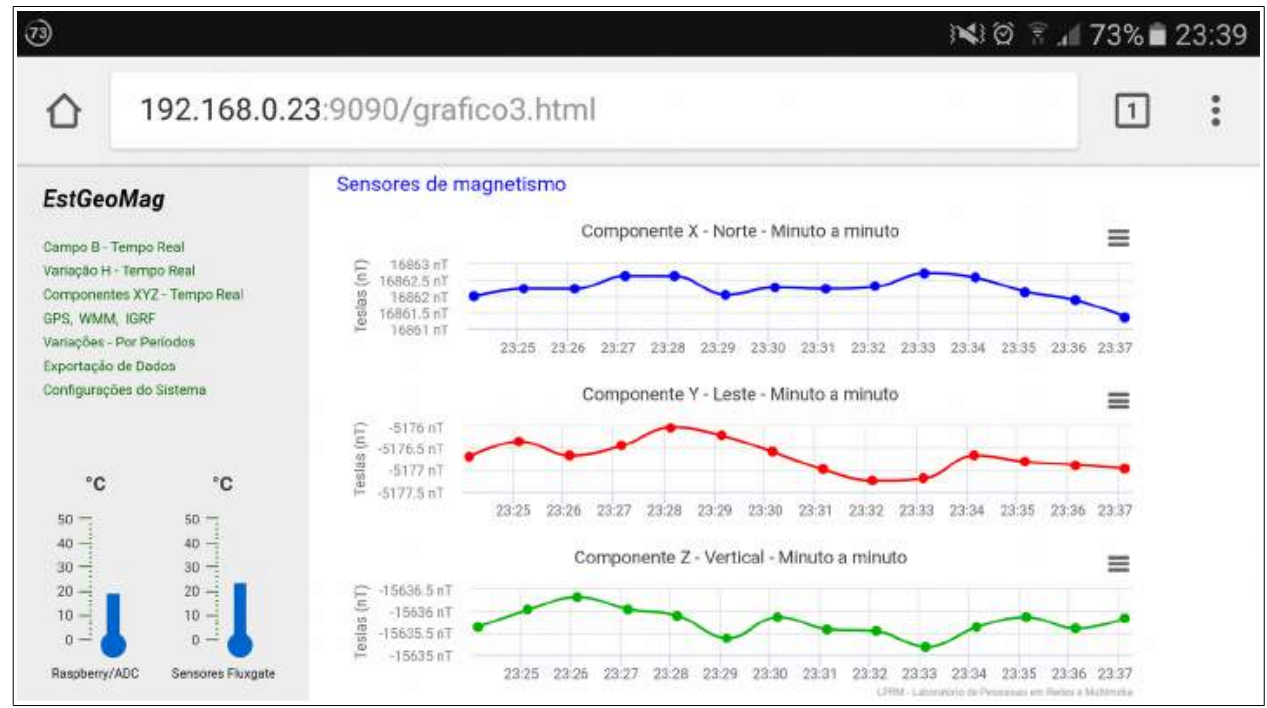

Figura 4: Interface Web em uso em um dispositivo móvel

\section{Avaliação e resultados}

Para a avaliação inicial do desempenho do EstGeoMag em termos das medidas de campo geomagnético obtidas, foi escolhido o modo programável de maior precisão e menor ruído no ADC de 24 bits: ganho de 1x de seu PGA (Programmable Gain Amplifier) e taxa de amostragem de até 2,5 amostras por segundo.

Para se obter estabilidade no processo e, principalmente, uma boa acurácia nas medições, foi imprescindível a escolha de um local apropriado para instalação do magnetômetro. A Figura 5 mostra a instalação da unidade de coleta e da unidade de processamento.

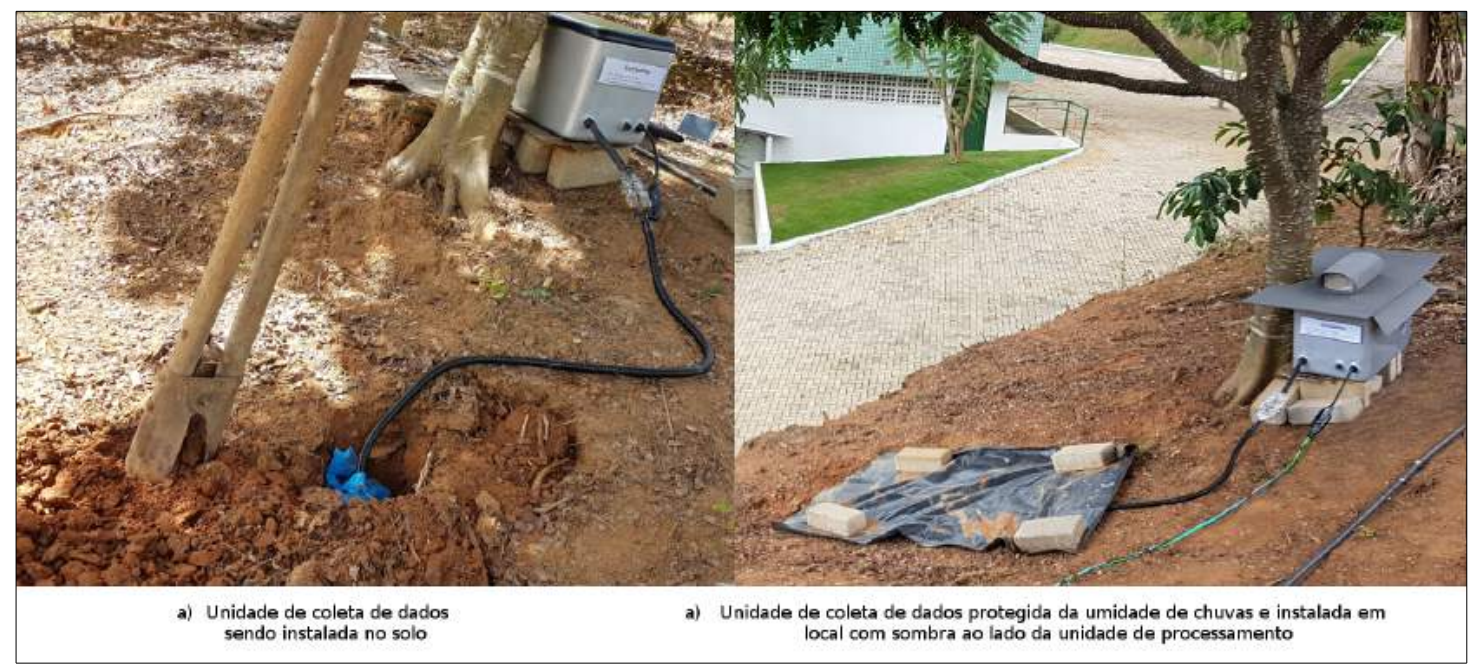

Figura 5: Instalação do equipamento 
Como ambos os sensores e o regulador de tensão da unidade de coleta são altamente sensíveis às variações de temperatura, a unidade foi enterrada no solo para garantir uma maior estabilidade térmica ao longo das variações climáticas diurnas e noturnas. Para ambas as unidades, o ideal é estarem em local com sombra e distante no mínimo de $20 \mathrm{~m}$ de qualquer objeto metálico ferromagnético, pedras ou rochas magnéticas, postes e linhas de energia elétrica ou ainda qualquer aparelho eletrônico que possa gerar algum tipo de campo eletromagnético.

A avaliação inicial do EstGeoMag foi feita a partir da comparação dos valores obtidos por ele com as medições realizadas no mesmo período por magnetômetros comerciais de alta precisão instalados nos seguintes locais:

- Petrolina, PE - mantido pelo Boston College e INPE, na rede $\mathrm{AMBER}^{4}$;

- Cachoeira Paulista, SP - mantido pelo INPE no programa EMBRACE ${ }^{5}$;

Apesar das diferenças dos valores de amplitude das medidas de campo magnético, as variações diurnas e noturnas nestes locais são muito semelhantes. De fato, para esse tipo de medida, as variações de campo observadas são, muitas vezes, bem mais importantes do que os valores absolutos do campo em si. Por exemplo, variações bruscas de campo em curtos espaços de tempo podem indicar a ocorrência de tempestades geomagnéticas.

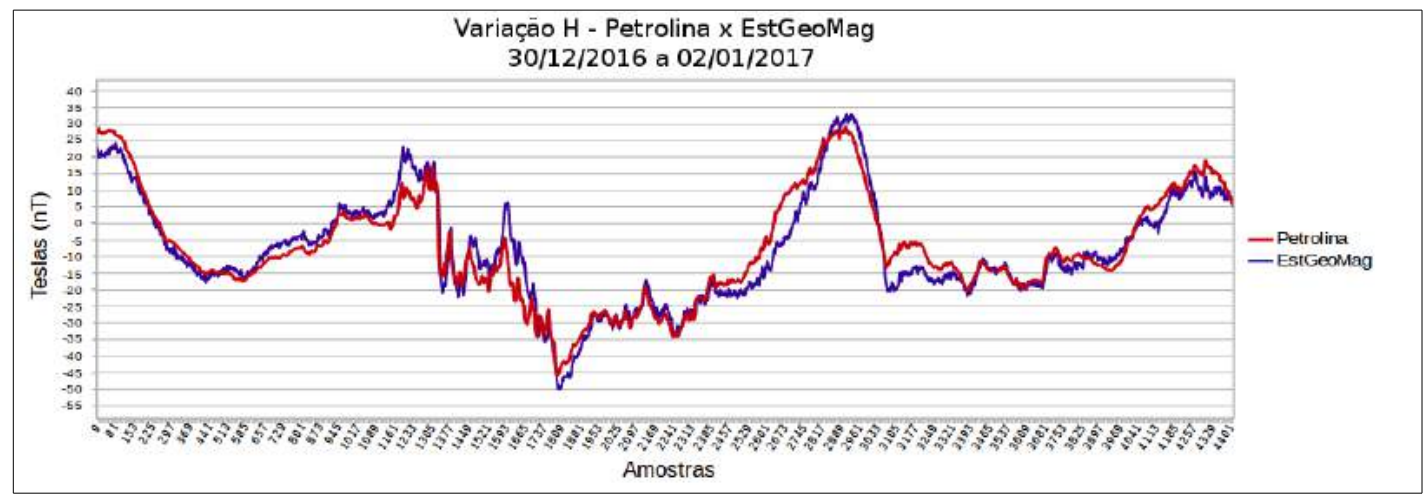

Figura 6: Comparativo entre o EstGeoMag e o magnetômetro da rede AMBER

Na Figura 6, podem ser vistos os comparativos de medidas entre o magnetômetro da Rede AMBER instalado em Petrolina - PE e o EstGeoMag instalado em Venda Nova do Imigrante - ES. O período escolhido para coleta dos resultados foi compreendido entre 30/12/2016 a 02/01/2017. Na mesma figura, é possível constatar que as poucas diferenças, nos momentos em que surgem, tendem a ser inferiores a $5 \mathrm{nT}$ dentre uma variação total diária média de $85 \mathrm{nT}$ pico a pico. O valor da diferença pode

4 http://magnetometers.bc.edu

5 http://www.inpe.br/climaespacial 
ser considerado baixo, considerando que entre os fatores causadores podemos citar o clima (e, portanto, a variação por temperatura causada nas medidas no local de instalação) e as diferenças topográficas e/ou geológicas das regiões, que são distantes cerca de $1500 \mathrm{~km}$ umas das outras.

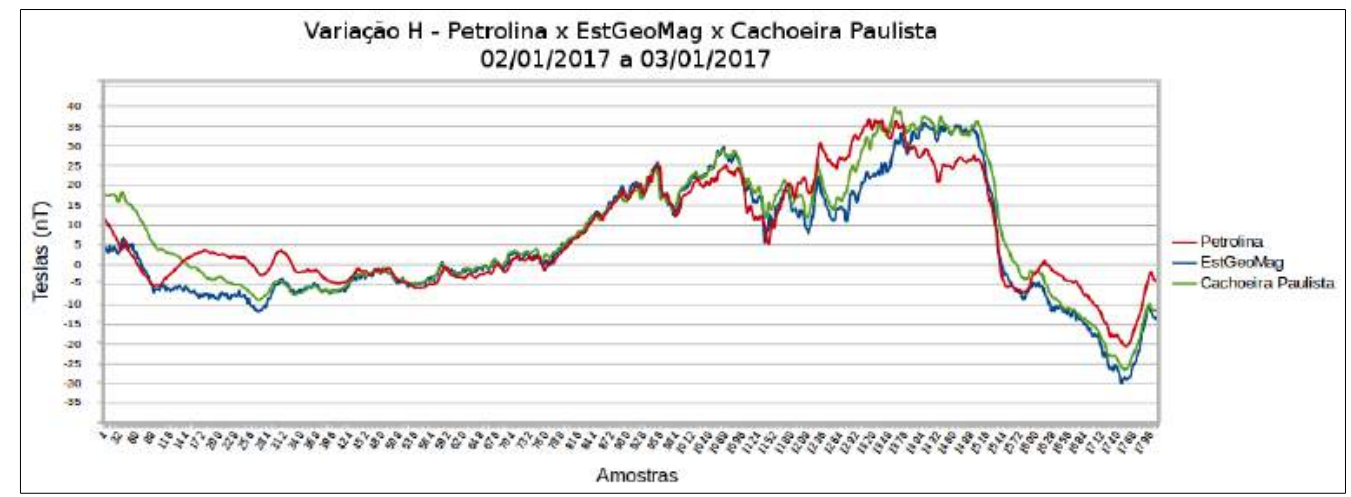

Figura 7: Comparativo entre o EstGeoMag e os magnetômetros do programa EMBRACE e da rede AMBER

A Figura 7 apresenta os comparativos entre os magnetômetros da Rede AMBER instalado em Petrolina - PE, do Programa EMBRACE instalado em Cachoeira Paulista - SP e o EstGeoMag instalado em Venda Nova do Imigrante - ES. O período escolhido para coleta dos resultados foi compreendido entre 02/01/2017 a 03/01/2017. É possível constatar que as leituras do EstGeoMag ficam bem próximas às realizadas pelos outros dois magnetômetros, em especial às do instalado em Cachoeira Paulista, situado a uma menor distância de Venda Nova do Imigrante: 600 km.

Analisando a Tabela 2, em intervalos de tempo dos dias 30/12/2016 a $01 / 01 / 2017$, podemos verificar que a diferença percentual entre as médias de desvio padrão das leituras de ambos magnetômetros é pequena, o que torna o EstGeoMag comparável aos magnetômetros do INPE.

Tabela 2. Comparativo de Desvio Padrão6

\begin{tabular}{|c|c|c|c|c|}
\hline \multicolumn{5}{|c|}{ Desvio Padrão - 30/12/16 a 01/01/17 } \\
\hline Magnetômetro & Data & Horário & Desvio Padrão (nT) & Diferença (\%) \\
\hline EstGeoMag & $30 / 12 / 16$ & $18 h-21 h$ & 5,0544 & 2.52775 \\
\hline AMBER & $30 / 12 / 16$ & $18 h-21 h$ & 5,1821 & $2,0<170$ \\
\hline EstGeoMag & $31 / 12 / 16$ & $12 \mathrm{~h}-15 \mathrm{~h}$ & 11,8193 & 3,15651 \\
\hline AMBER & $31 / 12 / 16$ & $12 h-15 h$ & 11,4462 & \\
\hline EstGeoMag & $01 / 01 / 17$ & $21 \mathrm{~h}-00 \mathrm{~h}$ & 2,7264 & 0,75608 \\
\hline AMBER & $01 / 01 / 17$ & $21 \mathrm{~h}-00 \mathrm{~h}$ & 2,7058 & \\
\hline Diferença média: & & & & 2,14678 \\
\hline
\end{tabular}

6 Os horários citados na Tabela 2 foram convertidos para o fuso horário UTC (Universal Time Coordinated, ou Tempo Universal Coordenado). O fuso horário típico da região onde o EstGeoMag está instalado é o UTC-3, porém na data das medidas já havia começado o horário de verão no Brasil, tornando-o portanto o fuso UTC-2. 


\section{Conclusões}

Podemos concluir que com medidas e desvio padrão equivalentes às dos magnetômetros comerciais utilizados pelo INPE, a solução de hardware e software desenvolvida e utilizada neste trabalho torna o EstGeoMag um magnetômetro adequado para acompanhamento das variações diárias do campo magnético terrestre. Sua arquitetura de hardware e software flexíveis e abertos, aliada ao uso de componentes de baixo custo permite a personalização da solução de acordo com as necessidades do usuário. $\mathrm{O}$ equipamento pode ser construído em larga escala para equipar escolas e universidades e auxiliar o ensino de geologia / geofísica para alunos de todo o país.

Como trabalhos futuros, podemos citar o aprimoramento do sistema de compensação por variação de temperatura, a inclusão de novos tipos de filtros lineares e não lineares para a redução de ruídos das medidas, o desenvolvimento de novas funcionalidades para a interface Web e a produção em escala da solução, visando observar as possíveis variações da precisão obtida em função dos componentes utilizados.

\section{Agradecimentos}

Este projeto teve financiamento parcial da FAPES, CAPES, CNPq e do projeto FUTEBOL (MCTIC/RNP). Gostaríamos de agradecer à equipe do INPE pelo dados obtidos e pela colaboração técnica e também ao Instituto Federal do Espírito Santo (IFES), campus Venda Nova do Imigrante, por ceder o espaço necessário para a instalação e realização dos testes do EstGeoMag.

\section{Referências}

Araujo, R. B., "Computação Ubíqua: Princípios, Tecnologias e Desafios". Livro de Minicursos do XXI Simpósio Brasileiro de Redes de Computadores, 2003. p 45-115.

CAMPBELL, W. H. Measurement methods. In: Introduction to geomagnetic fields. Cambridge University Press, 2003. p. 215-229

WANG, Jiabo; CHEN, Xi. A fluxgate magnetometer for navigation and sensing: noise character and digital filtering. In: SENSORS, 2015 IEEE. IEEE, 2015. p. 1-4.

OLIVEIRA et al. (2016, November). Uma Estação de Medição Geomagnética de Acesso Ubíquo para Estudos de Geofísica. In: Anais dos Workshops do Congresso Brasileiro de Informática na Educação (Vol. 5, No. 1, p. 1354).

SMITH, S. W. et al. Moving Average Filters. In: The scientist and engineer's guide to digital signal processing. 1997. p. 277-282.

BEGGAN, C. D.; MARPLE, S. R. Space weather goes to schools. Astronomy and Geophysics, v. 57, n. 2, p. 2.24-2.26, 2016.

SILVA JÚNIOR, J.P. Desenvolvimento de um magnetômetro fluxgate para estudos de clima espacial e aeroespacial. 2015. Dissertação de Mestrado. UFRN. 